\title{
El problema de la justificación del conocimiento básico
}

\author{
María Dolores García Arnaldos ${ }^{1}$
}

Recibido: 22 de enero de 2017 / Aceptado: 30 de abril de 2018

Resumen. El objeto de este artículo es analizar el problema de la justificación del conocimiento básico y ofrecer una solución basada en un tipo de justificación deflacionaria no-evidencialista a partir de la noción de habilitación (entitlement) de T. Burge y la de garantía racional de C. Wright. El problema, en el caso del conocimiento básico lógico, es que justificar las reglas lógicas inferencialmente supone utilizar principios lógicos, con lo cual se genera un círculo vicioso. Examinamos la viabilidad del enfoque no-inferencialista de Wright y sostendremos que una garantía racional es una respuesta viable para el problema de la justificación del conocimiento lógico básico.

Palabras clave: conocimiento básico; conocimiento lógico; habilitación; justificación inferencial; justificación no-inferencial.

\section{[en] The problem of the justification of basic knowledge}

\begin{abstract}
The purpose of this paper is to analyse the justification problem of the basic knowledge and offer a non-inferential solution based on a kind of deflacionary non-evidentialist justification from the notions of entitlement and rational warrant. The problem in the case of the basic logical knowledge, in general, is that to justify inferentially logical rules involves using logical principles, thus a vicious circle is generated. We examine the feasibility of Wright's non-inferentialist approach and we state that a rational warrant is a feasible answer to the problem of basic logical knowledge.
\end{abstract}

Key words: basic knowledge; entitlement; inferential justification; logical knowledge; non-inferential justification.

Sumario: 1. Introducción, 2. Conocimiento básico; 3. La justificación inferencial y la regresión al infinito; 4. Justificación epistémica "en sentido débil"; 5. Conclusión; 6. Referencias bibliográficas.

Cómo citar: García Arnaldos, M.D. (2019): "El problema de la justificación del conocimiento básico", en Revista Anales del Seminario de Historia de la Filosofía 36 (1), 243-259.

1 Universidad CEU-San Pablo (Madrid). PhD USC

dolores.arnaldos@rai.usc.es

ORCID: 0000-0001-9998-8060 


\section{Introducción}

El debate sobre si hay o no conocimiento básico parte del planteamiento inicial acerca de cuál es la naturaleza de un estado de conocimiento ${ }^{2}$ básico y cuál es la fuente de su justificación. En el segundo apartado presentamos algunos rasgos que caracterizan la noción de conocimiento básico con particular atención al conocimiento lógico básico. El problema en el caso de la lógica es que al justificar una regla de inferencia básica como el Modus Ponendo Ponens (MPP), ' $p$, si $p$ entonces $q$, luego $q$ ', inevitablemente nos vemos haciendo uso del mismo principio que queremos justificar. En términos generales, justificar las reglas lógicas inferencialmente supone utilizar principios lógicos, con lo cual se genera un círculo vicioso por comisión de petición de principio.

En la tercera sección se explican los rasgos de la justificación inferencial y los problemas que se le presentan a las teorías de la justificación entendidas en sentido estricto. A la luz del examen del tipo de justificación que se requiere para el conocimiento básico, se ha planteado la posibilidad de que la solución venga de la mano de una noción deflacionaria del concepto de justificación mismo. Así, en vez de 'justificación', algunos autores, entre los que está Burge (Burge, 1993, 2003), proponen nociones más débiles, 'habilitación'-entitlement- o 'garantía' -warrant(Pryor, 2005, pp. 182-183), con diferentes estatus epistémicos.

En la cuarta sección exponemos los rasgos de esta noción deflacionaria de justificación y analizamos, teniendo en cuenta las dimensiones internista y externista de la justificación, en qué medida posibilita y es compatible con el conocimiento. Además de Burge, Wright (Wright, 2004a, 2004b) ofrece una explicación alternativa a la justificación entendida en sentido estricto, que se presenta como adecuada, también, para el conocimiento lógico básico. Wright sostiene que dicha alternativa debe provenir de un tipo de justificación deflacionaria no-evidencialista. Este tipo de garantía racional es un enfoque no-inferencialista de la justificación que permite sortear los problemas de una justificación en sentido estricto y hablar de conocimiento básico.

\section{Conocimiento básico}

La distinción conocimiento básico/no-básico ${ }^{3}$ en epistemología contemporánea, se propone como respuesta a las tendencias escépticas presentes en la filosofía y como un modo de evitar paradojas, casos Gettier (Gettier, 1963) y el problema de la regresión al infinito. Aunque el problema se sitúa en el debate sobre la fundamentación del conocimiento que es tan antiguo como la misma filosofía ${ }^{4}$, la terminología 'conocimiento básico/no-básico' se ha comenzado a usar más habitualmente desde la segunda mitad del siglo $\mathrm{XX}^{5}$.

2 El conocimiento proposicional ha sido definido como creencia verdadera justificada (cf. Moser, 2002, p. 3). Se denomina tradicionalmente definición tripartita por contener tres cláusulas: alguien sabe que p: i) si $p$ es verdadero, ii) si, además, cree que $p$ y, iii) dicha creencia está justificada (cf. Dancy, 1993, p. 39). Autores contemporáneos que rechazan este planteamiento epistemológico son Quine (Quine 1969), Rorty (Rorty, 1979), Williamson (Williamson, 2000) y Kornblith (Kornblith, 2002) entre otros.

3 La distinción entre conocimiento básico/no-básico tiene su inspiración en A. Danto. "Freedom and Forebearance", 1965, pp. 45-63. Según Lehrer \& Paxson, 1969, p. 225, nota 1, Danto fue el primero en sugerirla.

4 En Aristóteles Segundos Analíticos A, 100b, 423 y Segundos Analíticos B, 19, 99.

5 En el Círculo de Viena, por ejemplo, encontramos los 'enunciados básicos o protocolares', que en este caso 
Hay autores, como Goldman (Goldman, 2012, p. 50), que tratan las nociones: 'básico', 'directo', 'inmediato' o 'fundacional', como sinónimos respecto de la justificación. Se podría definir el conocimiento básico como aquel que está justificado no-inferencialmente ${ }^{6}$. Los defensores de este tipo de justificación como R. Feldman (Feldman, 2003) y J. Pryor (Pryor, 2005), asumen que hay un modo directo de justificar una creencia sin que involucre a otras creencias o estados o proposiciones. Sin embargo, hay filósofos que no aceptan que haya una justificación inmediata y para otros es algo que no tiene ni siquiera sentido. Desde el coherentismo (BonJour, 1985; Davidson, 1986), por ejemplo, se niega que sea posible que algunas creencias estén justificadas de modo inmediato. El infinitismo, por otra parte, defendido por P. Klein (Klein, 1999), es una teoría en la que se rechaza que haya algo así como una justificación no-inferencial.

Entre los defensores de la justificación no-inferencial o inmediata se encuentran los que sostienen el fundacionismo. La directriz elemental de la concepción fundacionista es el considerar dos formas de justificación, la inferencial y la noinferencial. Nuestras creencias se justifican por la relación inferencial que tienen con otras creencias que están, a su vez, justificadas. Por ejemplo, al conocer un determinado axioma puedo inferir un determinado teorema. Si la justificación que tenemos para creer una proposición no se apoya en la justificación para creer otras proposiciones, entonces se trata de una justificación no-inferencial. El conocimiento inferencial, en cambio, es aquel que resulta de inferencias deductivas o inductivas desde unas proposiciones (eventualmente una) a otras proposiciones que sirven como evidencia o justificación. Para BonJour (BonJour, 1985), es claro que las creencias no-inferenciales son básicas ${ }^{7}$ respecto a la justificación en cuanto soporte de aquellas otras creencias (inferenciales, no-básicas) que dependen y derivan de ellas. Según el fundacionismo, las creencias justificadas no-inferencialmente son las basadas en experiencias o en intuiciones, y en cierto sentido, a ellas se accede de forma directa, automática. BonJour (BonJour, 1998, pp. 106-107), por ejemplo, sostiene que es posible un conocimiento directo e inmediato, se trata de la intuición racional (rational insight).

Hay un tipo de conocimiento directo, denominado knowledge by acquaintance (Russell, 1910-1911; 1912), es decir, conocimiento sin mediación alguna, directo o por familiaridad, que es inmediato, no interviene ningún proceso inferencial.

aluden al conocimiento básico empírico, es decir, observaciones experimentales particulares $(C f$. O. Neurath, "Protokollsätze", Erkenntnis 3, 1932-1933, 204-214. Vers. esp., "Proposiciones protocolares", en A. Ayer (ed.), 1965). Carnap emplea sobre todo la expresión 'enunciado protocolar' y Popper habla específicamente de 'enunciado básico’. Popper (Popper, 1972) usa la expresión conocimiento básico en un sentido más amplio. La distinción conocimiento básico/no-básico estaba implícita, pues, en los positivistas lógicos, particularmente en la discusión sobre la base del conocimiento empírico. Lo común en estos autores es la concepción fundacionista de la justificación. Aquí nos interesa la cuestión de en qué sentido el conocimiento es básico y qué lo distingue del conocimiento no-básico en el debate actual (a partir de la distinción de Danto y Lehrer), y especialmente cómo afecta al caso de la lógica.

6 'By 'non-inferential justification' (or, synonymously, 'foundational justification'), I mean justification of a belief which is not constituted by its being based on some other (justified) belief. Basing a belief on some other belief can constitute inferential justification of the resulting belief (if certain appropriate conditions are met). (...) In other words, non-inferential justification is the 'ultimate generation' or 'source' of justification, whereas inferential justification is the 'transmission' or 'extension' of justification. 'Foundationalism' (about justification) is the view that there are non-inferentially justified beliefs (sometimes called 'basic beliefs')." (Hofmann, 2012, pp. 2-3)

7 Entre los autores que no aceptan la posibilidad de creencias básicas, véase, por ejemplo, K. Lehrer, 1990. 
Es una relación cognitiva directa con un objeto $^{8}$, -que Russell identifica, por ejemplo, con ciertos particulares como nuestros datos sensoriales ${ }^{9}-$. Actualmente, si el conocimiento directo (by acquaintance) se puede considerar o no un tipo de conocimiento básico es algo que todavía se debate, según Fumerton (Fumerton, 2009).

El conocimiento básico, tanto el empírico como el lógico, ha llegado a representar un problema para la epistemología por las dificultades con las que nos encontramos a la hora de dar cuenta de su justificación. La dificultad que plantea el caso de la lógica frente al caso del conocimiento básico empírico se deriva del siguiente problema en el que consideramos dos aspectos. El primero es el problema de la justificación a priori, es decir, cómo damos cuenta de fuentes del conocimiento $a$ priori como la intuición, por ejemplo. Un presupuesto, pues, del que partimos es que hay conocimiento a priori.

En el ámbito de la lógica se distinguen dos áreas o dimensiones de aprioricidad; así, podemos decir que tenemos justificación a priori para creer leyes formales metalógicas y, por otra parte, que tenemos justificación a priori para creer e inferir verdades lógicas (Dogramaci, 2012); un ejemplo del primer tipo es la justificación para creer la validez del MPP, es decir, para creer que todo argumento que tenga la forma ' $p$, si $p$ entonces $q$, luego $q$ ', preserva la verdad. En cambio, tenemos una justificación a priori para creer e inferir verdades lógicas cuando inferimos usando, por ejemplo, el MPP; en este caso la justificación a priori se transmite de las premisas a la conclusión inferencialmente (Dogramaci, 2012, pp. 769-770). Que algún ámbito de la lógica se use para justificar otro no resulta problemático porque eso es precisamente el conocimiento inferencial ${ }^{10}$, el conocimiento que obtenemos cuando usamos la lógica ( $C f$. Hale, 2002, p. 279). Ahora bien, según Audi (Audi, 1998, p. 159), una inferencia no es una fuente básica de justificación o conocimiento, sino que más bien transmite y extiende la justificación y el conocimiento cuando se dan las circunstancias apropiadas, desde una o más premisas a la conclusión que se infiere de ellas. Así, sabemos que, si inferimos una conclusión de dos premisas verdaderas usando por ejemplo el MPP, nuestra conclusión será verdadera. A este tipo de conocimiento, de este u otros principios lógicos, le llamamos conocimiento lógico básico (Cf. Hale, 2002, p. 279).

8 Fumerton ("Classical Foundationalism" en De Paul, M. (ed.) 2001) y Plantinga ("Direct Acquaintance?" en De Paul, M. (ed.) 2001) también defienden la justificación de las creencias básicas en virtud de una relación o contacto directo (direct acquaintance). Esta relación de acquaintance para Fumerton es una cierta clase de acto mental o relación (De Paul (ed.), 2001, p. 61). Para Plantinga, la relación de acquaintance se extiende más quizás de lo que sugiere Fumerton.

9 (Cf. Russell, 1912, cap. V). Este problema fue motivo de debate en el Círculo de Viena en torno a las oraciones protocolares. Hasta principios de los años 30 para algunos positivistas, uno de los métodos para determinar el valor de verdad de un enunciado era comparando la sentencia evaluada con otra "básica" o "protocolar" que se asumía como definitivamente segura. Los enunciados básicos u observacionales constituían el punto de partida de la ciencia ( $c f$. Coffa, 1991, pp. 227; 249). La ciencia consistía, así pues, en la contrastación no con experiencias, sino con enunciados acerca de experiencias, observacionales o protocolares, lo cual proporcionaba una mayor intersubjetividad y para el Círculo de Viena esto era una ventaja. Incluso la lógica (aun no siendo empírica) estaba sujeta a revisión a partir de bases empíricas, como Carnap sostuvo en The Logical Syntax of Language. (Carnap, 1937, §82, p. 318; cf. Coffa, 1991, pp. 349-350). A principios de los años 30, Neurath puso en discusión que las oraciones protocolares fuesen enunciados que no era necesario justificar; también los enunciados protocolares son revisables, según Neurath, todas las creencias están sujetas a revisión y son falibles (cf. Coffa, 1991, p. 363).

10 Contemplamos el razonamiento deductivo, sin llegar a pronunciarnos sobre otros tipos de inferencias y sin llegar a cuestionar que esos modos de razonamiento también siguen reglas de inferencia específicos $(c f$. Schechter, forthcoming). 
Este es el segundo aspecto del problema y un punto controvertido; en lógica hay reglas de inferencia que se denominan básicas o primitivas, como el MPP, porque de ellas derivan otras, pero desde el punto de vista de la justificación y el conocimiento, no se consideran fuentes básicas. Por ejemplo, el MPP es una, entre otras, de las reglas básicas mediante las cuales caracterizamos la relación de consecuencia lógica proposicional clásica. La (meta)lógica para esta lógica, establece que es una regla de inferencia correcta (sound), que transmite la verdad. Además, desde el punto de vista epistemológico, como hemos dicho, es una regla de inferencia básica que transmite la justificación proporcionando conocimiento inferencial ${ }^{11}$. En ese sentido, la regla básica justifica inferencialmente el resto de reglas derivadas. Ahora bien, esta justificación no proviene de la regla del MPP, sino que la regla es el instrumento que proporciona conocimiento de la relación de consecuencia, de qué se sigue de qué y en qué consiste esa relación de consecuencia, siempre y cuando ese sea nuestro punto de partida. A las creencias inferenciales, así como al conocimiento y justificación inferencial se les denomina indirectas, precisamente porque únicamente las adquirimos a través de otras creencias, conocimiento o justificación.

El problema se presenta, pues, con reglas básicas como el MPP o con leyes como 'si p, entonces p': sabemos que el MPP, por ejemplo, es una regla válida, pero es difícil dar una explicación satisfactoria sobre el origen de ese conocimiento, de dónde proviene y cómo podría ser adquirido (Wright, 2004b, p. 155). Así, se trata de responder, entre otros, al problema de cómo podemos justificar, si podemos, los principios fundamentales de la lógica. Justificar este tipo de conocimiento resulta problemático, y algunos consideran que no puede ser justificado; esto se debe a que todo intento de demostración, prueba o justificación utiliza, y por tanto asume de partida, alguno de estos principios lógicos o todos ellos ( $C f$. Cohen \& Nagel, 1993, p. 187$)^{12}$.

\section{La justificación inferencial y la regresión al infinito}

El argumento que impulsó el debate de si hay o no justificación inmediata o noinferencial más conocido es el de la regresión al infinito. Dicho argumento tiene como fin demostrar que la justificación inferencial no es una auténtica justificación ya que siempre existe la amenaza de una regresión sin fin (Carroll, 1895). Como solución, las opciones que se presentan son, o bien explicar que dicha regresión no es problemática y por qué no lo es; o bien mostrar cómo detener esa regresión, o aceptar que la justificación inferencial es imposible. Aquí presentaremos algunas de las soluciones más conocidas, pero no son las únicas ${ }^{13}$.

11 Para un análisis de los problemas relativos a la transmisión de la justificación en una inferencia, cuestión en la que no entraremos, $c f$. Moretti, \& Piazza, 2013.

12 Véase también la crítica de Quine; a Carnap en su intento de justificar el conocimiento lógico (W.V. Quine. "Truth by Convention". En Quine, 1966).

13 Entre otros, ya en Popper se encuentra el problema de la relatividad de los enunciados básicos y una propuesta de solución al trilema de Fries ( $c f$. Popper, 1959, $2^{\text {a }}$ parte, cap. V, §29). Otra formulación análoga del problema es la del trilema que Diógenes Laercio atribuye a Agripa. El trilema de Agripa sostiene que no es posible justificación alguna, dadas las dificultades a las que se enfrentan tanto el fundacionismo, como el coherentismo y el infinitismo, como veremos a continuación. (Para una descripción más completa de las posibles soluciones cf. N. Rescher. Infinite Regress: The Theory and History of Varieties of Change. New York: Routledge, 2010. Agradezco esta referencia a uno/a de los evaluadores/as). 
Una de las teorías que defienden que hay justificación no-inferencial y ofrece una respuesta al problema de la regresión es el fundacionismo, precisamente, porque acepta que algunas creencias están justificadas de modo inmediato. La tarea principal de un fundacionista es, pues, explicar cómo se justifican las creencias básicas dado que actúan de 'generadoras' epistémicas.

Desde el coherentismo, en cambio, la justificación de las creencias depende de la coherencia dentro del sistema de creencias y siempre remite a otras creencias ${ }^{14}$. El argumento de Sellars (Sellars, 1963) contra la justificación no-inferencial fundacionista es que no podemos tener una justificación epistémica no-inferencial de una creencia porque sólo estamos justificados epistémicamente en sostener una creencia si tenemos una buena razón para pensar que la creencia es verdadera. Y tener una razón para pensar que una creencia es verdadera es ya una creencia, según Sellars (y BonJour); la justificación dependerá de la justificación de otras creencias, de modo que la justificación no-inferencial de creencias fundacionales es un mito. El problema es que los enfoques coherentistas priorizan las conexiones internas del sistema de creencias que son de tipo inferencial; por lo que, la justificación inferencial del conocimiento lógico básico no se presenta como la opción más prometedora ya que genera circularidad.

Una tercera respuesta al problema de la regresión es la que da el infinitismo epistémico $^{15}$. Desde esta perspectiva, el que haya una infinidad de creencias que tengamos que justificar para tener una creencia justificada inferencialmente no supone un problema y que se produzca una regresión al infinito de las justificaciones inferenciales tampoco afecta a la justificación la cual, a pesar de la regresión, se sigue considerando genuina. Puesto que desde el enfoque infinitista (Klein, 1999) una creencia justificada inferencialmente puede tener una infinidad de creencias que la justifiquen y se rechaza que haya algo así como una justificación no-inferencial, se supone que tenemos que ser capaces de operar con infinitas premisas en infinitos argumentos. No obstante, aun admitiendo la regresión de la justificación inferencial infinita, el problema del infinitismo es que habrá creencias que estén justificadas de un modo condicional, esto es, estarán justificadas si, y sólo si, las creencias en las que se basan están justificadas; creemos que tres es mayor que uno si, y sólo si, dos es mayor que uno y tres es mayor que dos, por ejemplo. Es decir, el problema es que lo que tenemos en cada eslabón de la cadena, es una creencia que de hecho no está justificada, sino que está justificada sólo de un modo condicional (Moser, Mulder \& Trout, 1998, p. 82). Así, esta tercera alternativa tampoco parece ser una opción que ofrezca soluciones viables al problema de la justificación del conocimiento lógico básico.

Un elemento común que tienen las propuestas anteriores es que conciben la justificación epistémica en sentido 'fuerte' o estricto. Según Conee y Feldman (Conee \& Feldman, 2004), la justificación epistémica se puede defender desde el evidencialismo $^{16}$ o desde el fiabilismo ${ }^{17}$. El evidencialismo epistémico sostiene

14 Para Davidson "nada puede contar como una razón para sostener una creencia excepto otra creencia" ( $c f$. Davidson, 1974 y, Davidson, 1984).

15 Hay una invitación al infinitismo epistémico por ejemplo ( $c f$. Peirce, 1931, §135, 56. También, Peirce, 1972).

16 El evidencialismo (E. Conee \& R. Feldman, 2004) sostiene que cualquier evidencia a favor de una creencia es inmediatamente una razón suficiente para aceptarla como verdadera (por lo menos a falta de otra más fuerte en contrario).

17 "What we call evidentialism is the view that the epistemic justification of a belief is determined by the quality 
que la meta, además de la verdad, es llegar a creerla por buenas razones. Según BonJour (BonJour, 1985), el núcleo de la idea de una justificación epistémica ${ }^{18}$ está relacionado con el ser epistémicamente responsables ${ }^{19}$ respecto de lo que uno cree y lo que la distingue de otros tipos de justificaciones es que tiene como meta cognitiva la verdad. La justificación, en este sentido, cumple un papel relevante por su vínculo con la actividad de la reflexión crítica. Además, la razón debe ser conclusiva, debe garantizar la verdad de la proposición en el sentido de que no sea posible que la proposición pudiese ser falsa desde esa razón (BonJour, 2002, p. 41).

El problema de una justificación epistémica entendida en sentido estricto es, precisamente, la cláusula que establece que la razón debe ser conclusiva. Esta concepción de la justificación que exige evidencias ${ }^{20} \mathrm{o}$ razones conclusivas nace de la epistemología cartesiana y de la idea de certeza como ausencia de duda alguna acerca de la corrección de las creencias (conocimiento indubitable).

Hay otras soluciones que parten de una concepción de conocimiento diferente como el contextualismo, teoría que se inspira sobre todo en Wittgenstein (On Certainty), según la cual el sujeto acepta o asume algunas proposiciones como puntos de partida de su indagación. Dichas proposiciones, ofrecen soporte a otras proposiciones y funcionan de este modo, como proposiciones básicas en un contexto determinado. Son proposiciones básicas que, a diferencia de las creencias justificadas no-inferencialmente, carecen de soporte evidencial (Moser, Mulder \& Trout, 1998, pp. 94-95). Es conocimiento básico, pero no evidencial. El problema es que estas creencias que no están justificadas (o proposiciones 'contextualmente' básicas) pueden llegar a justificar creencias falsas. El contextualismo (Williams, 2001) necesita explicar, pues, cómo una creencia sin base evidencial puede proporcionar justificación inferencial a otras creencias.

Esto es lo que BonJour denomina una concepción débil frente a la concepción fuerte del conocimiento (BonJour, 2010). La primera no exige razones o justificaciones conclusivas para el conocimiento, sino que, aunque no garantice necesariamente su verdad, es suficiente una justificación razonablemente fuerte. El problema es cuán probable debe ser la justificación para que sea creíble y, en consecuencia, en función de la 'fortaleza' o 'debilidad' de la justificación, evaluar si es compatible con poder realizar afirmaciones de conocimiento.

of the believer's evidence for the belief." (Conee \& Feldman, 2004, p. 83; p. 101).

18 "Epistemic justification of a proposition is evidence of the truth of the proposition." (Conee \& Feldman, 2004, p. 240)

19 "The idea of avoiding...irresponsibility, of being epistemically responsible in one's believings, is the core of the notion of epistemic justification." (BonJour, 1985, p. 8).

20 Otra concepción del conocimiento que no vamos a considerar aquí es la de Williamson (Williamson, 2000). Williamson defiende la ecuación evidencia = conocimiento donde es el conocimiento lo que justifica. Sin embargo, la postura de Williamson es excesivamente intelectualista, considera el conocimiento un estado y no una actividad (cf. Williamson, 2000, p. 199) y reduce el conocimiento práctico o saber-cómo a conocimiento proposicional o saber-que. Williamson se ve obligado a defender que todo conocimiento es proposicional ya que solo las proposiciones pueden ser razones para la creencia. No obstante, no es claro que resuelva los problemas que se le presentan a la factividad del conocimiento, es decir, cómo dilucidar entre un estado mental factivo y otro que no lo es, y el discernir la corrección del juicio correspondiente de conocimiento, considerando, sobre todo, el conocimiento lógico básico. 


\section{Justificación epistémica "en sentido débil"}

Dadas las dificultades con las que se encuentran estas teorías de la justificación epistémica, algunos filósofos han estudiado la posibilidad de algún tipo de justificación en sentido más 'débil': 'habilitación' o 'garantía', pero quizás con mayor alcance. Ahora bien, en qué medida una justificación en sentido 'débil'o 'habilitación' resulta más adecuada para la caracterización de la noción de conocimiento básico.

Burge presenta su teoría de la justificación entendida en sentido 'débil' (entitlement) a priori por primera vez en "Content Preservation" (Burge, 1993). Según Burge, la diferencia entre justificación y habilitación (entitlement) es que mientras que la justificación, entendida como una propiedad epistémica aplicada a capacidades reflexivas, necesita ser conceptualizada por el sujeto, el entitlement no. Se asemejan, en cambio, en que ambas tienen fuerza positiva como soporte racional de actitudes proposicionales o prácticas cognitivas y constituyen por ello un derecho epistémico. La habilitación es una suerte de derecho epistémico, una legitimación o una cierta garantía que, a diferencia de la justificación en sentido estricto, no necesita ser comprensible al sujeto, ni siquiera es necesario que sea accesible ni estar en el repertorio cognitivo del sujeto. Esta justificación en sentido débil está basada en el Principio de Aceptación ${ }^{21}$ y se deriva de que seamos agentes racionales (Burge, 1993, p. 467); ello sitúa a su teoría entre las teorías epistémicas por defecto: "Justified (entitled) acceptance is the epistemic 'default' position." (Burge, 1993, p. 468)

Según Burge, estamos racionalmente legitimados al razonamiento deductivo, así como a la percepción o la memoria, por ejemplo. La memoria o el razonamiento deductivo son fuentes racionales y por ello, fuentes de verdad prima facie. Lo que hace que una razón epistémica para creer algo sea auténtica es, precisamente, que aporte una base razonable para aceptar una creencia como verdadera (Burge, 1993, p. 470). Burge subraya el aspecto teleológico de la fuente racional (Burge, 2003, p. 507). Este aspecto teleológico, se comprende a través de la reflexión sobre lo que es la práctica racional y cuáles son sus funciones; una de las funciones primarias de la razón es la de presentar la verdad; y es por eso por lo que, en ausencia de razones contrarias, un sujeto está racionalmente legitimado (entitled) a confiar en el razonamiento deductivo o la memoria (Burge, 1993, p. 475).

La noción de legitimación es introducida por Burge como una forma de garantía debido a las consecuencias indeseables tanto del internismo como del externismo. Los internistas tienden a un híper-intelectualismo epistemológico, que exige la capacidad de reflexión crítica asociada a la responsabilidad epistémica. Por otra parte, los externistas niegan esta explicación y ponen el acento en indicadores fiables en el proceso de formación de la creencia externos al sujeto (Smithies, 2015). Para los externistas, que una creencia esté justificada no depende de una capacidad de reflexión crítica. El factor que hace verdadera una creencia y que epistémicamente la justifica, puede ser entera o parcialmente externo a la perspectiva cognitiva del sujeto. El fiabilismo de Goldman (Goldman, 1979) es una de las versiones del externismo más conocidas. Desde el fiabilismo, una creencia justificada es una creencia producida fiablemente; pero incorpora un factor causal a la justificación (Goldman, 1967). La dificultad de este fiabilismo causal, según Fumerton (Fumerton, 2001), es que no

21 "A person is entitled to accept as true something that is presented as true and that is intelligible to him, unless there are stronger reasons not to do so. Call this the Acceptance Principle.” (Burge, 1993, p. 467). 
explica cómo un sujeto puede tener una creencia que sea el resultado de un proceso poco fiable o nada fiable, de modo que no se le puede culpar o responsabilizar por el modo en el que se ha formado dicha creencia. A resultas de ello, no cabría decir que esa creencia no está justificada. Pero, la normatividad de la justificación tendría que ser algo posible si el conocimiento entendido como creencia verdadera da el derecho a estar seguros (Fumerton, 2001, p. 50).

Por su parte, Burge (Burge, 2003) desarrolla una teoría externista que se pueda aplicar a sujetos no-reflexivos, como los niños pequeños y evitar con ello la híperintelectualización en epistemología. Mientras que, en la justificación internista, los sujetos tienen que tener acceso a sus razones, las cuales deben de estar en el repertorio cognitivo del sujeto, para Burge, se puede estar epistémicamente legitimado a una creencia sin tener razones que garanticen la creencia, sin el repertorio conceptual necesario para tener razones relevantes para la creencia y sin tener los conceptos para comprender o pensar la habilitación. En definitiva, tener una razón o una justificación en este sentido, no requiere reflexión o comprensión, no es necesario que el sujeto tenga los conceptos necesarios para pensar el contenido proposicional de dicha garantía. Estas garantías epistémicas son a priori y son noevidentes, falibles, no demostrativas y no 'ciertas', según Burge, en el sentido de que las creencias justificadas a priori también están sujetas a revisión. En breve: "Entitlement is warrant without reason." (Burge, 2013, p. 490). Es clara la distancia entre este enfoque y el de las teorías de la justificación a priori 'fuertes' basadas en creencias 'directamente evidentes' (directly evident), o básicas a priori, según las cuales un tipo de conocimiento como el conocimiento a priori, es infalible.

En realidad, las concepciones contemporáneas del conocimiento a priori no asignan ya este rasgo de infalibilidad y la mayoría de las epistemologías contemporáneas manifiestan ser falibilistas (Hetherington, 2005). La cuestión es si hay alguna relación entre el carácter falibilista o no de la concepción del conocimiento y el que se postule la posibilidad del conocimiento básico a priori ${ }^{22}$. En este sentido, una distinción que nos parece significativa, es la que señala Hale (Hale, 2002) al final de su artículo "Basic Logical Knowledge" entre incorregibilidad e infalibilidad. La propuesta de Hale, que respaldamos, es que las creencias lógicas básicas son incorregibles, son "immune to rational doubt" (Hale, 2002, p. 304). Pero esto no obliga a sostener que tales creencias sean infalibles o que tengamos "an infalible method of arriving at true logical beliefs" (Hale, 2002, p. 304). Hale sostiene dos tesis importantes: en primer lugar, nosotros podemos saber que una regla es correcta únicamente si la corrección de la regla está más allá de toda duda racional. En segundo lugar, lo que nos habilita a una creencia lógica básica es que ésta es inmune a la duda racional, no que nuestro conocimiento o creencia justificada lo sea; esto nos da pie a una idea de justificación más débil que admita la falibilidad.

Por su parte, Wright (Wright, 2004a, 2004b) plantea una alternativa al dilema de elegir entre una epistemología fundacionista o anti-fundacionista (como el coherentismo o contextualismo) considerando la posibilidad de una garantía (warrant) no-evidencial. Para ello, propone una noción débil o deflacionaria de justificación no-inferencial, la de 'habilitación' (entitlement) como una forma de garantía racional que no exige evidencia de verdad y que permite hablar de conocimiento básico evitando tanto la circularidad como la regresión al infinito de las justificaciones.

22 Un análisis pormenorizado de lo que requiere una justificación a priori falible se encuentra en (Casullo, 2003, cap. 3, pp. 56-78). 
Wright (Wright, 1985) sostiene que el fundacionismo tradicional se reconoce por dos características: en primer lugar, debe contar con proposiciones que sean epistemológicamente básicas, es decir, "que el ser afirmadas verdaderas ha de generar algún tipo de presunción lógica de su verdad"23 (que no es lo mismo que afirmar que tales creencias se conciben como incorregibles). Tradicionalmente, como hemos dicho, a las proposiciones epistemológicamente básicas que se afirmaban como verdaderas, se les otorgaba el rango de infalibles y auto-evidentes, es decir, el fundamento de una proposición básica es ella misma. Este era un modo de explicar el carácter auto-justificativo de los axiomas lógicos. Ahora bien, Wright señala la diferencia entre la incorregibilidad y lo que clásicamente se denomina auto-evidencia. No es lo mismo decir que las creencias lógicas son auto-evidentes, que decir que son incorregibles o exentas de toda duda. Asimismo, como indicamos arriba, decir que las creencias lógicas básicas son incorregibles, inmunes a la duda racional (Hale, 2002, p. 304), no nos obliga a sostener que tales creencias sean infalibles. En segundo lugar, el fundacionismo sostiene que hay proposiciones que se consideran básicas respecto de otras proposiciones que se justifican a partir de éstas. El vínculo evidencial de este tipo de proposiciones no-básicas o inferenciales debe depender de hipótesis que se sostienen en referencia a creencias básicas únicamente y sin ningún otro tipo de presuposición.

La postura opuesta, el anti-fundacionismo, que Wright presenta a partir de M. Williams (Williams, 2001; 2005) afirma, en cambio, que ninguna de nuestras creencias es básica, sino que el sujeto acepta algunas proposiciones como puntos de partida de su indagación, y son estas proposiciones las que ofrecen soporte a otras. Las 'proposiciones de partida' funcionan como proposiciones básicas en un contexto determinado, aunque no tienen base evidencial. Desde este enfoque 'contextualista' (Williams, 1991, 2001) ${ }^{24}$ se asume que cualquier vínculo evidencial está mediado por enunciados empíricos que tienen un papel meramente hipotético en un contexto dado, y que, en un contexto diferente, otros enunciados empíricos pueden refutarlos o cancelarlos. El problema, como vimos, es que estas proposiciones 'contextualmente' básicas pueden llegar a justificar creencias falsas. El contextualismo tiene que dar cuenta de cómo una creencia sin base evidencial puede proporcionar justificación inferencial a otras creencias.

Así, nos encontramos, según Wright (Wright, 1985), con una imagen en la que hay que optar entre el anti-fundacionismo, que podría conducir a algún tipo de relativismo epistémico, que quedaría inerme ante la crítica escéptica, o el fundacionismo ${ }^{25}$, desde el cual tenemos evidencias de ciertas creencias solamente en base a proposiciones que asumimos sin comprobación (untested assumptions). En este último caso, habrá pues, que recurrir a fuentes básicas de conocimiento como la experiencia y la intuición. En el caso del contextualismo, será un tipo de conocimiento del que simplemente tenemos certeza y del que no tenemos evidencia ni fundamentos. Para

23 C. Wright. "Facts and Certainty", 1985. Reprinted in Th. Baldwin and T. Smiley (eds.). Studies in the Philosophy of Logic and Knowledge. Oxford: Oxford University Press, 2004, pp. 51-94, p. 84.

24 Cf. Rysiew, 2011. Notos anti-fundacionistas en el ámbito de la filosofía continental son F. Nietzsche y M. Foucault.

25 También hay opciones mixtas. Susan Haack (Haack, 1993) y Ernest Sosa (Sosa, 1991) entre otros, han buscado una alternativa intermedia entre el fundacionismo y el coherentismo que incorpore elementos de los dos. Una de las objeciones a este tipo de enfoques mixtos es que, en los niveles básicos, en definitiva, se sigue partiendo de un cierto fundacionismo (cf. Fumerton, 2002, pp. 230-231). 
Wittgenstein (Wittgenstein, 1969) se trata de un conocimiento no-inferencial para el que no es necesaria una explicación, porque no está basado en razones sino en la confianza. Si no dudamos, tampoco hay por qué explicarlo. Wright, en cambio, sí busca explicar cómo es posible el conocimiento no-inferencial, y su propuesta, a nuestro parecer, difiere de las anteriores. La idea de Wright es probar si el enfoque anti-fundacionista puede eludir los problemas de la justificación del conocimiento básico a partir de la consideración de que las conexiones evidenciales más básicas no necesitan justificación.

Wright (Wright, 1985, 2004a) sostiene que buena parte de nuestro razonamiento ordinario depende de presuposiciones. Esas presuposiciones no son proposiciones factuales, ni necesitan justificación epistémica; sin embargo, son fundamentales en nuestra indagación, hasta tal punto que no podemos prescindir de ellas. La garantía racional consiste en la habilitación a asumir las presuposiciones iniciales de un "Proyecto Cognitivo (PC)", siempre y cuando no haya evidencia en su contra, incluso careciendo de evidencia a su favor. De tal modo que, si el PC es valioso o indispensable, no contamos con información que ponga en duda la garantía de la presuposición e intentar verificar dicha presuposición nos llevaría a presuposiciones semejantes y no más seguras que esa, y así, sin un final, estamos habilitados a asumir la presuposición inicial del PC. Esta garantía consiste en un modo de aceptación de una proposición, y puede ser racional, pero no equivalente a creer (en el sentido importante de creencia). Como vemos, esta idea es muy semejante a la que encontramos en el Principio de Aceptación de Burge (nota 20).

A diferencia de Wittgenstein, en cambio, Wright distingue entre proposiciones 'especiales' (standing certainties) válidas en diferentes contextos y 'condiciones de autenticidad' de las que no tenemos evidencia, pero que no son válidas en diferentes contextos sino en cada ocasión cognitiva presente. Esto lo distancia de Wittgenstein y le permite salir al paso de los problemas que se le presentaban al contextualismo de Williams, ya que, aunque sea débilmente, se puede justificar el conocimiento a partir de una garantía no-evidencial.

Según Wright, las afirmaciones de conocimiento se basan en una aceptación previa de presuponer que mis capacidades cognitivas funcionan apropiadamente y también del ambiente cooperativo. De esas presuposiciones no hay evidencia (Wright, 2004b, p. 161). La ventaja sobre la justificación epistémica ('fuerte') es que la habilitación, la confianza racional en la que basarse, es suficiente en los casos donde no hay nada que justifique. La confianza racional como modo de aceptación de una estrategia unificada puede tener un alcance que no tiene la evidencia, lo cual supondría una ventaja explicativa.

Además de los dos casos anteriormente indicados: el funcionamiento adecuado de las facultades cognitivas relevantes y en el ambiente cooperativo, el tercer caso susceptible de garantía racional que Wright presenta son las reglas lógicas básicas. Esta propuesta de Wright, representa una solución al problema de la justificación del conocimiento lógico básico; es decir, sabemos que el MPP, por ejemplo, es una regla válida, pero es difícil dar una explicación satisfactoria sobre el origen de ese conocimiento, de dónde proviene y cómo podría ser adquirido (Wright, 2004b). La solución de Wright es la siguiente: a las reglas lógicas básicas se les otorga un papel presuposicional en un amplio rango de proyectos cognitivos y se les aplican consideraciones análogas a las aplicadas en el caso de las garantías de un PC (Wright, 2004b, p. 166). Este otro tipo de presuposición (o 'condición de autenticidad') es una 
habilitación racional, siempre que se cumplan los dos requisitos: que no encontremos razones para poner en duda las reglas lógicas básicas en cuestión y, en segundo lugar, que, si intentamos justificarlas, nos hallamos con reglas inferenciales del mismo tipo de las que queremos justificar ${ }^{26}$. Dado que, en principio, carecemos de evidencia de la invalidez de las reglas lógicas básicas y, mientras no encontremos dicha evidencia, estamos habilitados a aceptar su validez (Wright, 2004b, p. 166), podemos considerar las reglas de inferencia básicas ${ }^{27}$ dentro de los tipos de 'condiciones de autenticidad'. Ahora bien, ¿resulta suficiente para las reglas lógicas una habilitación o garantía racional? ¿Por qué confío en dichas reglas? ¿Por qué presupongo unas, como las reglas básicas, y no otras?

El problema de las inferencias básicas es su 'justificación'. Justificar una regla de inferencia comprende cierto conocimiento inferencial y en los casos básicos, dice Wright, parece inevitable depender de principios de inferencia en el meta-lenguaje del mismo tipo que el de la regla en cuestión (Wright, 2004b, p. 166). En este caso, sostiene Wright (Wright, 2001, p. 81), la solución no consiste en, por ejemplo, apelar a la validez del MPP añadiendo información nueva. Por el contrario, el problema se resuelve no-inferencialmente, de modo directo, con un tipo de garantía noinferencial. Si no queremos sostener que la aprehensión de la validez de las reglas lógicas básicas es algo intuitivo, la opción de Wright es clasificarlas como garantías de proyectos cognitivos. Estamos habilitados a confiar en la corrección de 'a basic inferential apparatus' (Wright, 2004a, p. 208). El problema es, dice Wright, darnos cuenta de si las reglas lógicas (por ejemplo, la del condicional) son correctas. En este caso, la cuestión es que el contenido del condicional viene dado por la práctica de la inferencia de acuerdo al MPP y a la prueba condicional, y tenemos esa práctica o la información, por la que sería reconocido intuitivamente correcto (Wright, 2004b, p. 171). Pero, se pueden dar diversos errores tales como la inconsistencia en una práctica determinada, y la posible inclinación a decir que sabemos que nuestra práctica (en el caso del condicional) no cae en esos errores, de modo que, si es que los cometemos, no depende del MPP (como en el caso McGee (cf. McGee, 1985)).

Para Wright, pues, la opción para afirmar que sabemos que el MPP es correcto es afirmarlo como un entitlement generalizado de un proyecto cognitivo (Wright, 2004b, p. 172). Ahora bien, ¿es posible que sólo contemos con una 'habilitación' para justificar la validez de las reglas básicas de inferencia? Para responder, tendríamos que examinar primero otros tipos de justificación con el objetivo de encontrar uno que permita sortear tanto la circularidad como la regresión al infinito, examen en el que aquí no podemos entrar ${ }^{28}$.

26 Burge señala en una nota que la comprensión de una verdad lógica simple produce justificación porque la fuerza justificacional para el caso de la lógica deriva del propio contenido de la verdad lógica (Burge, 1993, p. 480, nota 19).

27 Naturalmente, es necesario explicar qué entendemos por una inferencia básica y que esta definición evite caer de nuevo en la circularidad o la regresión al infinito. Esto es posible si se entiende la inferencia básica como una acción mental básica, como Wright (Wrigth, 2014) y García-Arnaldos (García-Arnaldos, 2017) abordan en otro lugar.

28 En el caso del conocimiento lógico, para que la justificación evite estos dos problemas, dicha justificación debe provenir o bien de una fuente independiente (como la intuición, BonJour, 1998; Bealer, 2000) o de un tipo de justificación deflacionaria, que no conlleve dar razones (en sentido fuerte, razones como evidencias) como hemos visto, es el caso de una garantía racional (Wright 2004b). También sería el caso del 'razonamiento ciego' (Boghossian, 2003) en el que aquí no entramos (cf. García-Arnaldos, 2016). 
La ventaja de la propuesta de Wright es que es un tipo de garantía a priori noinferencial que permite salir al paso a las dificultades que tienen las justificaciones 'por defecto'. Por ejemplo, en el caso del conocimiento lógico, según Burge: "Being competent to make a modus ponens inference does not require a capacity to think schematic generalizations or to think about propositional contents or logical constants -much less a capacity to think the rule and use it to justify a transition. Entitlement to rely on such transitions does not require justifications that cite the rules that codify and explain the transitions. They do not even require a capacity to cite the premises as a reason for the conclusion." (Burge, 2013, p. 492). Así entendido, una persona podría ser competente al inferir sin tener la capacidad siquiera de aportar las premisas como razón para la conclusión que se deriva de ellas. A mi juicio, esta explicación pone en duda la noción misma de inferencia, pues no se entiende en qué consiste entonces un razonamiento. Las garantías 'por defecto', además, no llegan a explicar cómo es que hay creencias que en virtud de ellas mismas y sin origen epistémico alguno, están justificadas correctamente (Wright, 2001). Tampoco explican cuáles son las creencias que caen bajo esta caracterización y cuáles no. No son la mejor opción para defender la objetividad de la lógica.

Desde mi punto de vista, tener una cierta competencia inferencial requiere cuando menos la capacidad de realizar una acción mental básica como es la del razonamiento y la capacidad de dar las premisas como razones para la conclusión (Autor, 2017). Esas capacidades se pueden llevar a cabo sin saber que se trata de la regla del modus ponens y sin saber que dicha regla justifica la corrección de mi razonamiento. No es necesario 'saber que se sabe' para ser competentes al inferir. En cualquier caso, nuestro objetivo, como el de Wright, no es el de suplantar la justificación por una habilitación, sino cualificar nuestro conocimiento o juicio de orden superior, de modo que sea posible salir del impasse al que conduce el debate entre fundacionismo/anti-fundacionismo. Así pues, la concepción alternativa del tipo de justificación que se requiere para el conocimiento básico, en la que tiene cabida una noción deflacionaria de este concepto, ha de entenderse como una ampliación de la noción de justificación, nunca como licencia para reemplazarla.

\section{Conclusión}

Al abordar el problema de la justificación del conocimiento básico hemos considerado diferentes respuestas al argumento de la regresión al infinito, una de ellas es el contextualismo (Wittgenstein, Williams) desde el que se sostiene que el sujeto asume algunas proposiciones como puntos de partida $\mathrm{y}$, aunque no tienen base evidencial, ofrecen soporte a otras proposiciones funcionando, de este modo, como proposiciones básicas en un contexto determinado. El problema del contextualismo es explicar cómo una creencia sin base evidencial puede proporcionar justificación inferencial a otras creencias. Hay además algunos modos de entender la justificación epistémica que se consideran fuertemente evidencialistas. Si justificación se entiende en sentido fuerte, evidencialista, entonces tendremos que recurrir a fuentes básicas de conocimiento, por ejemplo, la intuición o la experiencia. Cabe hablar de una noción más débil de justificación como la de Burge. La justificación que propone Burge es una sub-especie de garantía epistémicamente externista, precisamente porque no requiere que el sujeto tenga los conceptos necesarios para pensar el contenido 
proposicional de dicha garantía. No obstante, antes de cualquier posibilidad de justificación, aquello que se nos presenta como verdadero debe ser comprensible, inteligible. Burge parte de dicha inteligibilidad como elemento necesario y previo de toda posible justificación; la inteligibilidad a priori es, pues, un signo de racionalidad prima facie (Burge, 1993, p. 488). Además, Burge subraya el aspecto teleológico de la fuente racional. Este aspecto se comprende a través de la reflexión sobre lo que es la práctica racional y cuáles son sus funciones; una de las funciones primarias de la razón es la de presentar la verdad ${ }^{29}$; y es por eso por lo que, en ausencia de razones contrarias, un sujeto está racionalmente legitimado a confiar en el razonamiento deductivo o la memoria (Burge, 1993, p. 475).

Por otra parte, Wright ha planteado una alternativa al dilema de elegir entre una epistemología fundacionista o anti-fundacionista considerando la posibilidad de una garantía no-evidencial. Para ello, ha propuesto la confianza como modo de aceptación de una estrategia unificada, ya que tiene un alcance que no tiene la evidencia. Su propuesta ofrece una teoría deflacionaria de la justificación que permite sortear además del escepticismo, la circularidad y la regresión al infinito. Además, puede complementar y salir al paso a los problemas que se le presentaban al contextualismo.

Tanto Burge como Wright toman la noción de 'habilitación' como una subclase dentro de las garantías epistémicas, una garantía racional no-evidencial. Sin embargo, mientras para Burge, dicha habilitación es un tipo de justificación por defecto, la propuesta de Wright se desmarca y presenta el modo de distinguir aquellas proposiciones a las que estamos habilitados, de las que no.

La ventaja, además, de la garantía racional sobre la justificación epistémica entendida en sentido estricto es que, en los casos donde no hay evidencia o no hay nada que justifique o demuestre el éxito cognitivo, una habilitación en la que basarse es suficiente. Uno de los tipos de garantía racional es el del 'aparato inferencial básico'. También en este caso, la ‘habilitación' es una forma de garantía que permite postular una teoría de la justificación no-inferencial, además de evitar caer en la regresión al infinito ${ }^{30}$.

\section{Referencias bibliográficas}

Aristóteles. Tratados de Lógica (Organon). vol. 2: Sobre la interpretación; Analíticos primeros; Analíticos segundos, Madrid: Gredos, D.L. 1995.

Audi, R. Epistemology. A contemporary introduction to the theory of knowledge. London: Routledge, 1998.

Ayer, A. (ed.). El positivismo lógico. México: F.C.E., 1965.

Bealer, G. "A Theory of the A priori”. Pacific Philosophical Quarterly. vol. 81, 2000, pp. 1-30.

29 "Reason has a function in providing guidance to truth, in presenting and promoting truth (...)" (Burge, 1993, p. 475)

30 Quisiera agradecer a los profesores Concha Martínez, Xavier de Donato, José Miguel Sagüillo, Javier Vilanova, María Caamaño, M. José Frápolli y Víctor Verdejo por sus sugerencias y propuestas de mejora para este trabajo. En particular a Concha Martínez por su generosidad en el constante seguimiento y la posibilidad de discusión de estos temas en innumerables ocasiones. A todos ellos, a los profesores José Luis Falguera, Uxía Rivas y Juan Sánchez del mismo departamento y a muchas personas que me han animado a seguir adelante a pesar de todo, agradezco su apoyo y confianza. 
Boghossian, P. A. "Blind Reasoning”. Proceedings of the Aristotelian Society, Supl., vol. 77, 2003, pp. 225-48. También en P. Boghossian, Content and Justification: Philosophical Papers. Oxford: Oxford University Press, 2008, pp. 250-267.

Bonjour, L. The Structure of Empirical Knowledge. Cambridge: Harvard University Press, 1985.

Bonjour, L. In Defense on Pure Reason: a Rationalist Account of A Priori Justification. Cambridge: Cambridge University Press, 1998.

Bonjour, L. Epistemology. Classic Problems and Contemporary Responses. Lanham, MD: Rowman \& Littlefield, 2002. $2^{\text {nd }}$ ed. 2010.

Bonjour, L. "The Myth of Knowledge”. Philosophical Perspectives, 24, 2010, pp. 57-83.

Burge, T. "Content Preservation". The Philosophical Review, 102, 1993, pp. 457-488.

Burge, T. "Perceptual Entitlement". Philosophy and Phenomenological Research, 68 (3), 2003, pp. 503-548.

Burge, T. Cognition through understanding. Oxford: Oxford University Press, 2013.

Carnap, R. Logische Syntax der Sprache. 1934. (English translation: The Logical Syntax of Language, New York: Humanities, 1937).

Carroll, L. "What the tortoise said to Achilles". Mind, vol. 4, 14, 1895, pp. 278-280.

Casullo, A. A Priori Justification. Oxford: Oxford University Press, 2003.

Coffa, J. A. The semantic tradition from Kant to Carnap. To the Vienna Station. Cambridge: Cambridge University Press, 1991.

Cohen, Morris R., \& Nagel, E. An Introduction to Logic. Ed. by John Corcoran. Indianapolis: Hackett, 1993.

Conee, E. \& Feldman, R. "Evidentialism”. Earl Conee \& Richard Feldman, Evidentialism: Essays in Epistemology, Oxford: OUP, 2004.

Dancy, J. Introducción a la epistemología contemporánea. Madrid: Tecnos, 1993.

Danto, A. "Freedom and Forebearance". Freedom and Determinism, New York: Random House, 1965, pp. 45-63.

Davidson, D. "On the Very Idea of a Conceptual Scheme". Proceedings and Addresses of the American Philosophical Association, 47, 1974.

Davidson, D. Inquiries into Truth and Interpretation. Oxford: Clarendon Press, 1984.

Davidson, D. "A Coherence Theory of Truth and Knowledge", In E. Lepore (ed.) Truth and Interpretation: Perspectives on the Philosophy of Donald Davidson. Oxford: Blackwell Publishing, 1986.

De Paul, M. (ed.). Resurrecting Old Fashioned Foundationalism. Lanham. Rowman and Littlefield, 2001.

Dogramaci, S. “Apriority”. D. G. Fara, \& G. Russell, The Routledge Companion to Philosophy of Language, New York: Routledge, 2012, pp. 768-782.

Feldman, R. Epistemology, Upper Saddle River, NJ: Prentice Hall, 2003.

Fumerton, R. “Classical Foundationalism”. En De Paul, M. (ed.), 2001.

Fumerton, R. "Theories of Justification". Paul K. Moser (ed.), The Oxford Handbook of Epistemology, Oxford: Oxford University Press, 2002, pp. 204-233.

Fumerton, R. "Knowledge by Acquaintance vs. Description”, The Stanford Encyclopedia of Philosophy (Summer 2009 Edition), E. N. Zalta (ed.), URL $=<$ http://plato.stanford.edu/ archives/sum2009/entries/knowledge-acquaindescrip/>. 2009, Consultado: 05/01/2013

Garcia-Arnaldos, M. D. "Una justificación no-inferencial de las inferencias básicas". In Eighth Meeting of the Spanish Society for Analytic Philosophy. SEFA. Oviedo: KRK Ediciones, 2016, pp. 79-86. ISBN: 978-84-8367-547-2. 
Garcia-Arnaldos, M. D. "Elizabeth Anscombe: razones y acciones”. In Ma Gloria Ríos Guardiola, Ma Belén Hernández Glez., E. Esteban Bernabé (eds.). Mujeres con luz. Murcia: Editorial EDITUM, colección Campus Mare Nostrum, 2017, pp. 89-108. ISBN 978-84-17157-41-8.

Gettier, E. “Is Justified True Belief Knowledge?”. Analysis. 23, 1963, pp. 121-123.

Goldman, A. I. “A Causal Theory of Knowing”. Journal of Philosophy, 64, 1967, pp. 357-372.

Goldman, A. I. "What is Justified Belief?". George S. Pappas (ed.). Justification and Knowledge, Dordrecht: Reidel, 1979.

Goldman, A. I. Reliabilism and Contemporary Epistemology: Essays. Oxford: OUP, 2012.

Haack, S. Evidence and Inquiry. Oxford: Blackwell, 1993.

Hale, B. "Basic Logical Knowledge". Royal Institute of Philosophy Supplement, vol. 51, Logic, Thought and Language, 2002, pp. 279-304.

Hetherington, S. "Fallibilism". 2005. Recuperado el 14 de 09 de 2012, de Internet Encyclopedia of Philosophy: http://www.iep.utm.edu/fallibil/

Hofmann, F. "Three kinds of reliabilism". Philosophical Explorations: An International Journal for the Philosophy of Mind and Action, 2012, pp. 1-22. DOI:10.1080/13869795 .2013 .738305

Klein, P. "Human Knowledge and the Infinite Regress of Reasons". Philosophical Perspectives. vol. 13, 1999, pp. 297-325.

Kornblith, H. Knowledge and its Place in Nature. Oxford: Oxford University Press, 2002.

Lehrer, K., \& Paxson, T. J. "Knowledge: Undefeated Justified True Belief'. The Journal of Philosophy, vol. 66, nº 8, 1969, pp. 225-237.

Lehrer, K. Theory of Knowledge. Boulder: Westview Press,1990.

Mcgee, V. “A Counterexample to Modus Ponens". Journal of Philosophy, 82, 1985, pp. 462471.

Moretti, L. \& Piazza, T. "Transmission of Justification and Warrant", The Stanford Encyclopedia of Philosophy (Winter 2013 Edition), Edward N. Zalta (ed.), URL = http:// plato.stanford.edu/archives/win2013/entries/transmission-justification-warrant/.

Moser, P. K. “A priori”. P. K. Moser, The Oxford Handbook of Epistemology. Oxford: OUP, 2002.

Moser, P. K.; Mulder, D. H.; Trout, J. D. The Theory of Knowledge. A Thematic Introduction. Oxford: Oxford University Press, 1998.

Peirce, C. S. "The first rule of reason", Collected Papers, vol. 1, C. Hartshorne \& P. Weiss (eds.), Cambridge, MA: Harvard University Press, 1931.

Peirce, C. S. The Essential Writings. E. C. Moore (ed.) New York: Harper \& Row, 1972. Reimp. Prometheus Books, 1998

Popper, K. R. The Logic of Scientific Discovery. London: Hutchinson \& Co, 1959.

Popper, K. R. Objective Knowledge. An Evolutionary Approach. Oxford: Clarendon Press, 1972.

Pryor, J. "There is Immediate Justification". In Matthias Steup \& Ernest Sosa (eds.). Contemporary Debates in Epistemology. Wiley-Blackwell, 2005, pp. 181-202.

Quine, W.V. "Truth by Convention”. En W. Quine. The Ways of Paradox and Other Essays, New York: Random House, 1966; ( $2^{a}$ ed. Cambridge, Mass.: Harvard University Press, 1976).

Quine, W.V. Ontological Relativity and Other Essays. New York: Columbia University Press, 1969. 
Rorty, R. Philosophy and the Mirror of Nature. Princeton, NJ: Princeton University Press, 1979.

Russell, B. "Knowledge by Acquaintance and Knowledge by Description". PAS New Series, Vol. XI, 1910-1911, pp. 108-128.

Russell, B. Problems of Philosophy. Oxford: Oxford University Press, 1912.

Rysiew, P. "Epistemic Contextualism". The Stanford Encyclopedia of Philosophy (Winter 2011 Edition), Edward N. Zalta (ed.), URL $=<$ http://plato.stanford.edu/archives/win2011/ entries/contextualism-epistemology/>.

Schechter, J. "Deductive Reasoning", The Encyclopedia of the Mind. edited by Hal Pashler, SAGE Publishing, forthcoming.

Sellars, W. "Empiricism and the Philosophy of Mind". Science, Perception and Reality, London: Routledge \& Kegan Paul, 1963.

Smithies, D. "Why Justification Matters". In David K. Henderson-John Greco, Epistemic Evaluation: Purposeful Epistemology. Oxford: OUP, 2015. DOI:10.1093/acprof:o so/9780199642632.003.0010

Sosa, E. Knowledge in Perspective. Cambridge: Cambridge University Press, 1991.

Sosa, E. \& M. Steup. Contemporary Debates in Epistemology. Cambridge: MA, Blackwell Publishers, 2005.

Williams, M. Unnatural Doubts: Epistemological Realism and the Basis of Skepticism. Cambridge, MA: Blackwell, 1991.

Williams, M. Problems of knowledge. A Critical Introduction to Epistemology. Oxford: OUP, 2001.

Williams, M. "Doing without Immediate Justification". M. Steup \& E. Sosa (ed.). Contemporary Debates in Epistemology. Oxford: Blackwell, 2005, pp. 202-16.

Williamson, T. Knowledge and its limits. Oxford: Oxford University press, 2000.

Wittgenstein, L. On Certainty. Ed. G. E. M. Anscombe \& G. H. von Wright. Trad. G. E. M Anscombe \& Denis Paul. Oxford: Blackwell, 1969.

Wright, C. "Facts and Certainty", Proceedings of the British Academy. 71, 1985, pp. 429-72. Reprinted in Th. Baldwin and T. Smiley (eds.) Studies in the Philosophy of Logic and Knowledge. Oxford: Oxford University Press, 2004, pp. 51-94,.

Wright, C. “On Basic Logical Knowledge”. Philosophical Studies, 106, 2001, pp. 41-85.

Wright, C. "Warrant for nothing (and foundations for free)?". Aristotelian Society Supplementary, vol. 78 (1), 2004a, pp. 167-212.

Wright, C. "Intuition, Entitlement and the Epistemology of Logical Laws". Dialectica, 58, 2004b. pp. 155-175.

Wright, C. "Comments on Paul Boghossian, 'What Is Inference?"”. Philosophical Studies, 169, 2014, pp. 27-37. 